CORRECTION

https://doi.org/10.1038/s41586-019-1214-3

\section{Author Correction: Evolutionary history resolves global organization of root functional traits}

Zeqing Ma, Dali Guo, Xingliang Xu, Mingzhen Lu, Richard D. Bardgett, David M. Eissenstat, M. Luke McCormack \& Lars O. Hedin

Correction to: Nature https://doi.org/10.1038/nature25783, published online 21 February 2018.

We thank reader Joseph Craine for pointing out three inadvertent errors in this Letter. First, 4 of the 71 divergence dates extracted from ref. ${ }^{1}$ of this Amendment and used in Fig. $1 \mathrm{~b}$ of the original Letter were overestimated. The correct values are 45 million years ago (Ma) for Apocynaceae, $51 \mathrm{Ma}$ for Anacardiaceae, $40 \mathrm{Ma}$ for Primulaceae, and $53 \mathrm{Ma}$ for Amaryllidaceae. These errors had little influence on the overall trend of Fig. $1 \mathrm{~b}\left(r^{2}\right.$ is now 0.48 rather than 0.54 , with no change to $P<0.001$ ) and do not change our conclusion and inferences. Second, we neglected to note that since refs. ${ }^{2}$ and ${ }^{1}$ of this Amendment considered only angiosperms, our Fig. $1 \mathrm{~b}$ necessarily did not include gymnosperm taxa. The in-text reference to Fig. $1 \mathrm{~b}$ should therefore read "all major angiosperm plant families in our dataset" rather than "all major vascular plant families in our dataset". Third, in Fig. 1c the trait value of mycorrhizal colonization for Machilus kwangtungensis was erroneously given the value 0.25 instead of 1.0. This error had little influence on the overall Fig. 1c trend, reducing $r^{2}$ from 0.64 to 0.63 (with no change to $P<0.001$ ).

In the Methods, we neglected to note that the divergence time for Magnoliaceae was taken from the recent analysis of ref. ${ }^{2}$. In addition, the Methods description should have noted that we excluded one specific root length (SRL) value (for Vaccinium mandarinorum) because its inferred tissue density $\left(1.96 \mathrm{~g} \mathrm{~cm}^{-3}\right)$ implausibly exceeded a value that was twice as dense as water and close to the density of rock.

In addition, there were five typographical errors in the Extended Data display items. (1) In Extended Data Fig. 2c, a tick and its corresponding tick label $\left(10^{\circ}\right)$ was missing from the $y$ axis. (2) In Extended Data Fig. 4 b, $r^{2}$ should be 0.06 instead of 0.07. (3) In Extended Data Fig. $5 a$, the letters indicating significance should be ' $b$ ' instead of ' $a b$ ' for the temperate biome, and 'abc' instead of 'ab' for the boreal biome. (4) In Extended Data Fig. 6h, the $x$-axis unit should be milligrams (mg) rather than micrograms $(\mu \mathrm{g})$ of nitrogen per gram of root per hour $\left(\mathrm{mg} \mathrm{N} \mathrm{g}^{-1}\right.$ root $\mathrm{h}^{-1}$ ). (5) In Extended Data Table 3, the column variable names 'RootN' and 'RootC' should be swapped.

Although the principal component analysis was not central to our main findings, we also now provide the abundances of species of different growth forms (woody versus herbaceous) and species from different biomes in Extended Data Table 2. In 'Main analysis', there were 104 woody and 0 herbaceous species; and 31 Tropical, 64 Subtropical and 9 Temperate biome species. In 'Excl.MC', there were 201 woody and 16 herbaceous species; and 39 Tropical, 118 Subtropical, 44 Temperate, 15 Desert and 1 Boreal biome species. In 'Gapped.MC.data', there were 201 woody and 16 herbaceous species; and 39 Tropical, 118 Subtropical, 44 Temperate, 15 Desert and 1 Boreal biome species. In 'Gap-filled', there were 281 woody and 88 herbaceous species; and 46 Tropical, 137 Subtropical, 104 Temperate, 14 Mediterranean, 53 Grassland, 24 Desert and 3 Boreal biome species. In the 'Gap-filled' analysis, the biome species count $(n=381)$ exceeds the total species number $(n=369)$ because several species occurred in more than one biome. None of these errors has been corrected online. Dali Guo is deceased.

1. Wikstrom, N., Savolainen, V. \& Chase, M. W. Evolution of the angiosperms: calibrating the family tree. Proc. R. Soc. Lond. B. 268, 2211-2220 (2001).

2. Massoni, J., Couvreur, T. L. \& Sauquet, H. Five major shifts of diversification through the long evolutionary history of Magnoliidae (angiosperms). BMC Evol. Biol. 15, 49 (2015). 